\title{
Pseudo-pericardial tamponade after perforation of the right coronary artery
}

\section{Steinwender, R Hofmann, F Leisch}

Heart 2004;90:e36 (http://www.heartjnl.com/cgi/content/full/90/6/e36). doi: 10.1136/hrt.2004.035378

A case of perforation of the right coronary artery, which was complicated by an intramural right ventricular haematoma with pseudo-pericardial tamponade resulting in fatal asystole, is presented.

A ccidental coronary artery perforation with a guidewire during percutaneous intervention is a complication with a wide range of possible consequences. Asymptomatic courses are also seen as acute haemodynamic deteriorations because of pericardial tamponade with compression of the right ventricle. ${ }^{12}$

We report on a case of perforation of the right coronary artery which was complicated by an intramural right ventricular haematoma with pseudo-pericardial tamponade, resulting in asystole.

\section{CASE REPORT}

A 71 year old man was hospitalised for evaluation of suspected coronary artery disease. He reported a two day long episode of typical chest pain at rest three weeks previously, followed by strictly exercise dependent angina pectoris. Co-morbidities included arterial hypertension and permanent atrial fibrillation. An ECG showed normal frequency atrial fibrillation but no sign of acute or remote ischaemia or Q waves. Cardiac enzymes including creatine kinase, its myocardial band (MB) fraction, and the transaminases were normal, troponin $\mathrm{T}$ was elevated $(0.4 \mathrm{ng} / \mathrm{ml})$.

Cardiac catheterisation demonstrated normal left ventricular function; coronary artery angiography revealed a $90 \%$ stenosis of the left anterior descending (LAD) branch of the left coronary artery combined with severe sclerosis of the small circumflex branch.

The right coronary artery (RCA) was occluded near the ostium with its widespread peripheral branches being fed by collaterals from the left coronary artery.

Considering the patient's history and the absence of diaphragm akinesia it was decided to attempt an interventional revascularisation of the RCA before stenting the LAD.

An ACS Hi-Torque Intermediate guidewire (Guidant, Indianapolis, USA) was chosen for the planned revascularisation.

As it was repeatedly impossible to cross the entire lesion with the wire, it was retracted from a position about $3 \mathrm{~mm}$ distal to the beginning of the occlusion. Angiography after wire retraction demonstrated a small depot of contrast medium evolving around the occluded vessel segment. No distinct perforation or distribution of contrast medium in the pericardial space was observed. A control angiography 10 minutes later displayed the same scene with the contrast medium depot slightly more pronounced (fig 1). The intervention was stopped at this time. The nearly asymptomatic patient was transferred to the cardiac care unit with

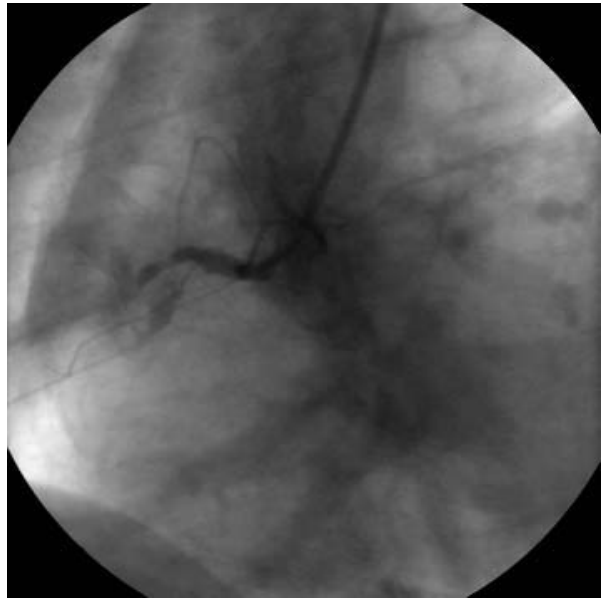

Figure 1 Small depot of contrast medium around the occlusion site of the RCA after guidewire retraction.

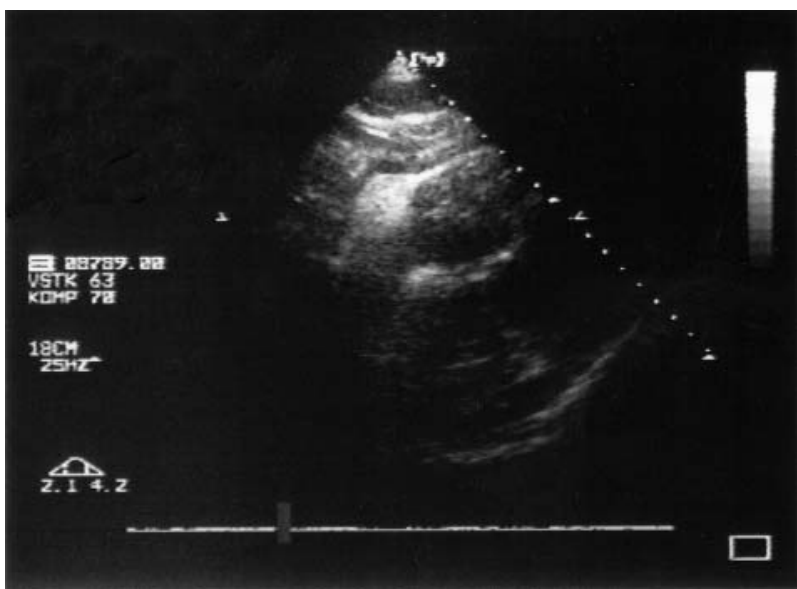

Figure 2 Intramural right ventricular haematoma $1.7 \mathrm{~cm}$ in diameter in the echocardiographic subcostal view.

stable haemodynamics (blood pressure 125/80 $\mathrm{mm} \mathrm{Hg}$, heart rate $75 /$ minute). Echocardiography showed no pericardial effusion with good right and left ventricular function, the ECG displayed no signs of ischaemia. Analgesia was initiated and heparin, which is routinely administered before interventions, was balanced by protamin.

During the following hour the patient's blood pressure and heart rate were stable, followed by a rapid haemodynamic deterioration with a drop of blood pressure to $70 / 50 \mathrm{~mm} \mathrm{Hg}$ and an increase of heart rate to 95 / minute over the next 15 minutes. The repeated echocardiography revealed a moderate pericardial effusion on the four chamber view and an 
intramural right ventricular haematoma of about $1.7 \mathrm{~cm}$ diameter in the subcostal view (fig 2). Severe compression of the right ventricle was not evident at this moment. Subxiphoid pericardiocentesis was attempted but could not be performed successfully. As haemodynamic stabilisation was not achieved by volume supply, urgent surgical management was intended. In transit to the operating theatre sudden cardiac arrest occurred because of asystole, requiring prolonged cardiopulmonary resuscitation. Asystole did not resolve after digital pericardial fenestration before surgery, but did so immediately following sternotomy, establishing stable haemodynamics.

No significant pericardial effusion was found. Further exploration revealed an intramural right ventricular haematoma with a diameter of about $5 \mathrm{~cm}$ at the site of the coronary perforation, which had compressed the right ventricular cavity. Thoracotomy had relieved the right ventricle, restoring sufficient blood circulation.

After coronary artery bypass grafting of the LAD and the RCA, the haemodynamically stable patient was transferred to the intensive care unit. Unfortunately he died some days later from severe hypoxic brain damage caused by the prolonged resuscitation. Necropsy showed good function of the bypass grafts and located the intramural right ventricular haematoma near the proximal RCA; a distinct perforation site could not be found.

\section{DISCUSSION}

To our knowledge this is the first reported case of an intramural right ventricular haematoma imitating the symptoms and haemodynamic consequences of a pericardial tamponade.

Coronary artery perforations can occur, especially when attempting to re-open occluded vessels, but this complication can usually be handled by experienced cardiologists themselves. ${ }^{3}$ This case demonstrates that an intramural haematoma compressing the right ventricle can provoke a fatal pseudo-tamponade in the absence of a significant pericardial effusion. The haemodynamic effects of the well known pericardial tamponade and the intramural pseudotamponade are the same; however, therapeutic consequences differ significantly.

Haemodynamic deterioration after perforation of the RCA can be caused by an intramural right ventricular haematoma, especially when no significant pericardial effusion is seen at echocardiography. Surgical intervention should be planned early, as cardiopulmonary resuscitation seems to be less effective because of the notably compressed right ventricle.

\section{Authors' affiliations \\ C Steinwender, R Hofmann, F Leisch, Cardiovascular Division, City Hospital Linz, Austria}

Correspondence to: Dr Clemens Steinwender, Cardiovascular Division, City Hospital Linz, Krankenhausstraße 9, A-4020 Linz, Austria; clemens.steinwender@akh.linz.at

Accepted 17 February 2004

\section{REFERENCES}

1 Owens PE, Motwani JG. Conservative management of contained coronary perforation after percutaneous intervention. Catheter Cardiovasc Interv 2002;57:215-6.

2 Altman F, Yazdanfar S, Wertheimer J, et al. Cardiac tamponade following perforation of the left anterior descending coronary system during percutaneous transluminal coronary angioplasty: successful treatment by pericardial drainage. Am Heart $J$ 1986;111:1196-7.

3 Gunning MG, Williams IL, Jewitt DE, et al. Coronary artery perforation during percutaneous interventions - incidence and outcome. Heart 2002;88:495-8.

4 Fejka M, Dixon SR, Safian RD, et al. Diagnosis, management, and clinical outcome of cardiac tamponade complicating percutaneous coronary interventions. Am J Cardiol 2002;90:1183-6. 